\title{
Development of MATLAB-Based Digital Signal Processing Teaching Module with Graphical User Interface Environment for Nigerian University
}

\author{
Oyetunji S.A*, Ale D.T \\ Federal University of Technology Akure, Nigeria \\ *Corresponding author E-mail: samlove98ng@yahoo.com, danielale9291@gmail.com
}

\begin{abstract}
The development of a teaching aid module for digital Signal processing (DSP) for Nigerian Universities was undertaken to address the problem associated with non-availability instructional module. This paper annexes the potential of Peripheral Interface Controllers (PICs) with MATLAB resources to develop a PIC-based system with graphic user interface environment suitable for data acquisition and signal processing. The module accepts data from three different sources: real time acquisition, pre-recorded data acquisition and software-generated data. The developed system is able to perform analysis on both stationary and non-stationary signals using Fast Fourier Transform (FFT) and Short Time Fourier Transform (STFT). The STFT-based approach provides Improved Joint Time Frequency Analysis (IJTFA) of signals. A graphical user interface (GUI) was developed to create interactive environment for understanding the operations of digital signal processing. The developed system not only provides interactive platform for data acquisition, spectral analysis and filter design but also makes it avoidable for students in the University.
\end{abstract}

Keywords: Data Acquisition, Embedded system, Filter, GUI and Signal processing

\section{Introduction}

The understanding of DSP at the undergraduate level can be enhanced with the development board. However, the inability of students from the developing countries like Nigeria to afford this teaching aid makes the subject less attractive. From the market survey, the cost ranges between $\$ 500$ and above $\$ 1000$ [1]. The importance of DSP in daily living with applications found in virtually all human endeavors from toys to cars and many others emphasis the need to acquire relevant knowledge of DSP. The areas of applications of signal processing include audio processing, data compression, robotic vision, radar and missile lunching. In addition advances in technology in recent years have placed the demand for accurate and precise interpretation of data and results on signals and data analysts. This is noticeable in weather forecast and flood control where satellite images are matched an in the application of DSP in crime detections using correlation theorem [2]. DSP module is useful for characterization of sensors in industry which is aimed at addressing the problems in the industries which are associated with lack of systems to estimate the performance of sensors over time. The estimation of power supply quality is made possible by such module by the Utility. These applications show that information is now better handled by DSP and places higher demand on the University products. A typical data acquisition system [3,4] consists of hardware, sensors and actuators, transducers, signal conditioning hardware, computer and application specific software. Fig. 1 shows the block diagram typical data acquisition system.

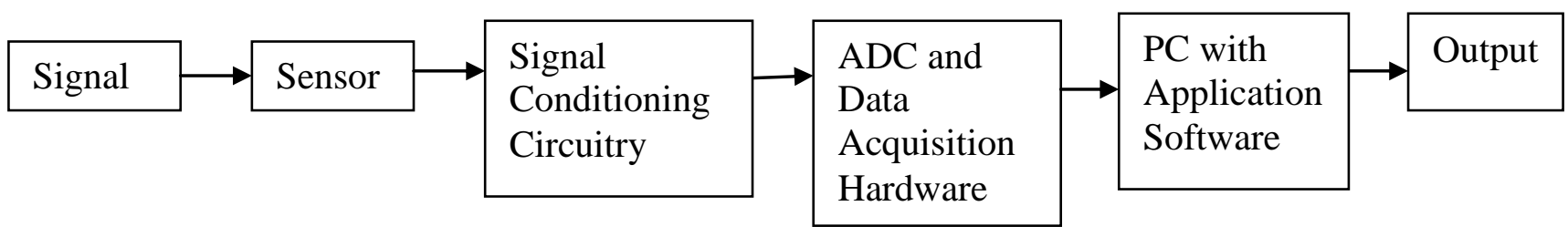

Fig 1: Data Acquisition Setup 
The data acquisition hardware is an embedded system which is usually built around a PIC microcontroller, Atmel microcontroller and ARM processor [4, 9]. Endowing PIC-based projects with GUI tools can speed the development process in data driven applications such as feedback control, smart sensors, etc. PIC microcontrollers include serial communication functionality to facilitate data communication with external devices such as analog-to-digital converters (ADC), 1-wire sensors, etc. The features considered in the choice of PIC include:
a) Eight channels ADC
b) Five digital ports
c) USART communication protocol supported
d) Memory capacity

In this work, PIC16F877A was used. The decision was based on cost and availability. It is a 40-pin embedded system with 8-bit CMOS FLASH dual inline package IC to facilitate serial communication between PIC and PC [5, 6]. The serial communication was made possible with RS232 driver/receiver connected to the microcontroller [6]. Similarly, MATLAB [8], commercially available interactive mathematical programming software, provides serial data communication functionality on PCs. In addition, SIMULINK, MATLAB's interactive icon-based programming environment, enables users to simulate and analyze dynamic system models. This platform was employed to develop an interactive GUI environment for PIC projects which provides enhanced data processing and visualization.

The effectiveness of MATLAB-based GUI environment to interact with PIC microcontroller projects is demonstrated by using three examples:

(a) import user data and save it into a location on C drive from the GUI;

(b) import signals from a sensor interfaced to the PIC into a the developed GUI; and

(c) use the GUI to analyze and process data.

The module was built on a MATLAB-based Graphical User Interface for PIC Microcontroller-Based Data Acquisition and Signal Processing in-line with the undergraduate curriculum in DSP in Nigeria University. The features include data Acquisition, spectral analysis, filtering, correlation and convolution and other relevant areas of applications. The analysis of non-stationary signals was handled by STFT-based approach which provides Improved Joint Time Frequency Analysis (IJTFA) of signals. A graphical user interface (GUI) was developed in MATLAB to create interactive environment for understanding the operations of digital signal processing. The cost of the module makes it avoidable for students in Nigeria University.

\section{Implementation of DSP Module based on the Curriculum}

The module was developed in line with the curriculum for undergraduate program in DSP as approved by National University Commission [10].

\subsection{DSP Undergraduate Curriculum in Nigeria University}

The course content for DSP in Nigeria University as approved by National University Commission (NUC) [10] includes Digital acquisition, Fourier Transform (DFT, FFT), Z Transform and Applications-Difference Equation, Filter, Correlation and Convolution and Multi-rate DSP.

\subsection{Hardware Environment}

PIC stands for "peripheral interface controller". It is of RISC (Reduced Instruction Set Computer) design based on PIC is Harvard architecture which is simpler and faster than other architectures. The hardware environment for this paper consists of a PIC microcontroller, a PC, a RS232 driver/receiver, and a DB-9 serial cable [7]. The PIC microcontroller is interfaced with external devices such as sensors (e.g., a light dependent resistor) and display (e.g., liquid crystal display). In addition, the PIC microcontroller performs embedded computing. The PC is used to write user specified embedded programs to be executed by the PIC microcontroller. Furthermore, the PC hosts an interactive GUI for the user to manipulate control variables and visualize sensory data. The PIC microcontroller and the PC communicate using a serial interface. It has a separate program and data bus. PIC16F877A is 16-bit device is available in 40-pin and 44-pin. It has 10 bit 8 channel, 3timer, and EPROM. The crystal oscillator used is a $20 \mathrm{MHz}$ type with two $22 \mathrm{pF}$ capacitors [1,9]. For the serial communication purpose, USART protocol was employed. The controller operates in 5V DC supply. 
The embedded system which performs analog to digital conversion for the analog input signal, logs the data and transfers serially to the PC where further analysis is done. The signal processing is performed on the data where further analysis will be performed on the signal. The microcontroller performs ADC operation on the signal using the 10 bits mode. A 16X2 liquid crystal display was incorporated into the work to enable proper monitoring of processes at the hardware level. The signal conditioning is done using a voltage follower, Level shifter and bandlimiting. The simulation of the hardware was done using ISIS. The design specification is as follows:

i) Input Signal:
a) Analogue 0-5V DC
b) Analogue $\mathrm{AC} \pm 2.5 \mathrm{~V}$
c) Input frequency $\leq 10 \mathrm{kHz}$
d) Digital 0-5V DC

ii) Output signals: Limited to the PC audio card specification

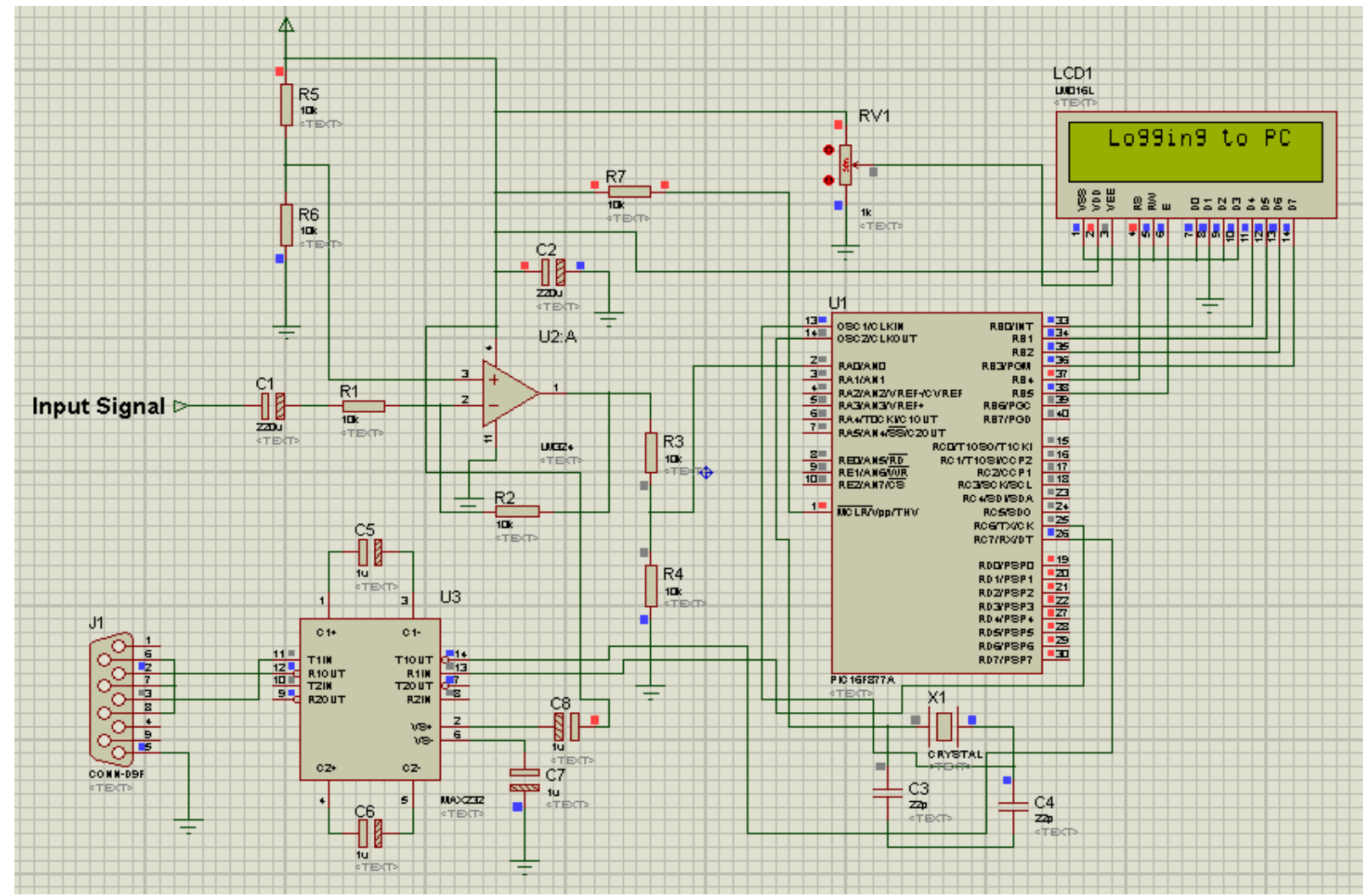

Fig 2: Data Acquisition Hardware

\subsection{Low Pass Filter}

Fig. 3 shows a second order Low pass filter using a second order Sallen and Key circuit diagram to bandlimit the signal before acquisition. This is necessary to obey Nyquist criteria and avoid aliasing. The filter is a two-pole active filter Butterworth filter.

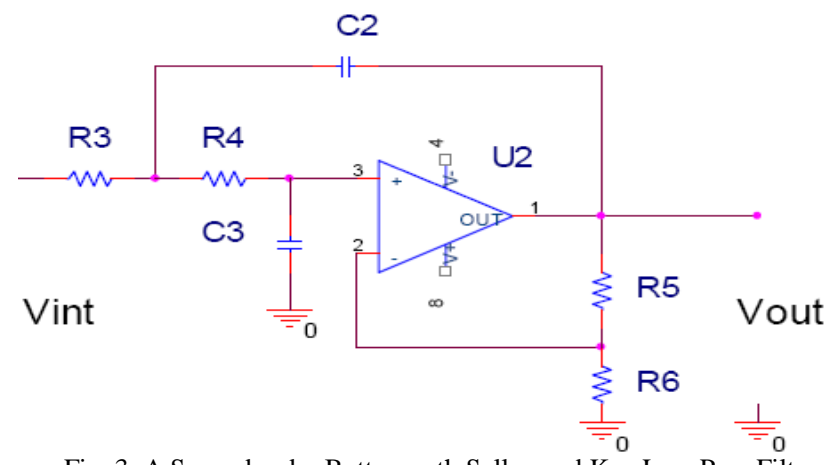

Fig. 3: A Second order Butterworth Sallen and Key Low Pass Filter 


\subsection{Development and Implementation of GUI}

The first interface (also known as the home screen) has a built-in signal generator, with which the amplitude, frequency and phase of a desired waveform can be set; it also features a noise generator which has a model for additive white Gaussian noise (AWGN) and some other noise models. A dual axis is then used to display the original signal and the noisy signal appropriately as shown in Fig. 4. This signal can be saved and exported to disk from the GUI. There are three sources of signals into this environment, these are live acquisition from embedded application hardware, importing of pre-recorded signal from disk and software-generated signal

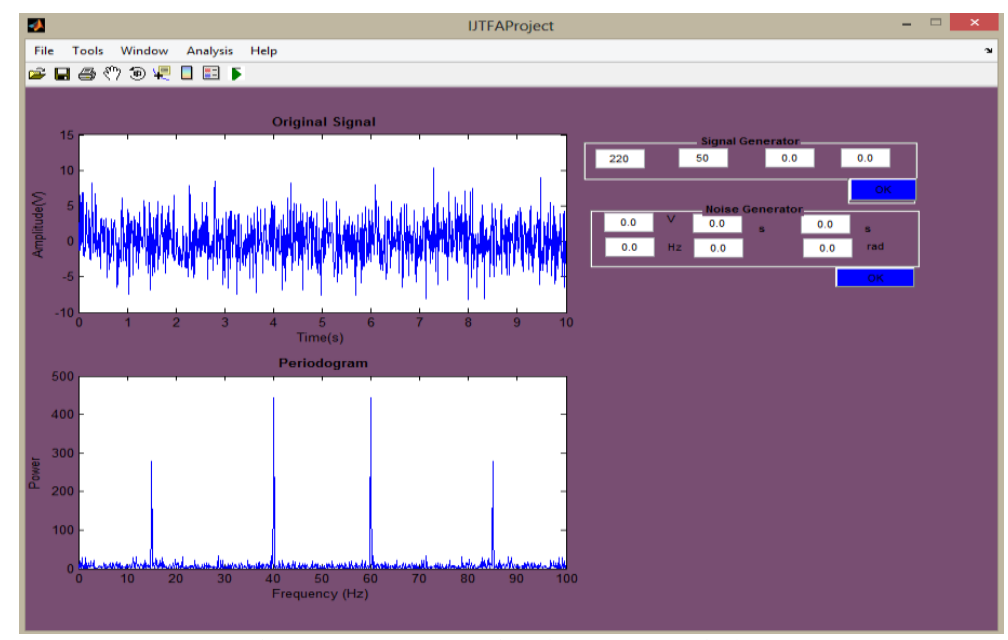

Fig. 4: Home Screen/Signal Generator Window

\section{Experimentation with the DSP Module}

The module was used to perform experiment in Digital Signal Processing in line with the objective of this research work which focuses on the development of DSP module suitable for the teaching of DSP in Nigeria University. The experiments include Data acquisition, signal analysis, filtering, Operation on recorded data and correlation.

\subsection{Data Acquisition}

The module accepts signals which are digital or analogue from two possible sources that are either internal or external to the module. The module accepts signals based on the input specification. The data acquisition consists of bandlimiting analogue filter and PIC 18F4550 microcontroller. The analogue filter was based on a two pole Sallen and Key Butterworth filter.

Data Acquisition into the system is made possible through serial communication from an embedded system, data is transferred asynchronously from the hardware to a user-specified location on the PC, which is readable and writeable from external processes. The developed window is shown in Fig. 5.

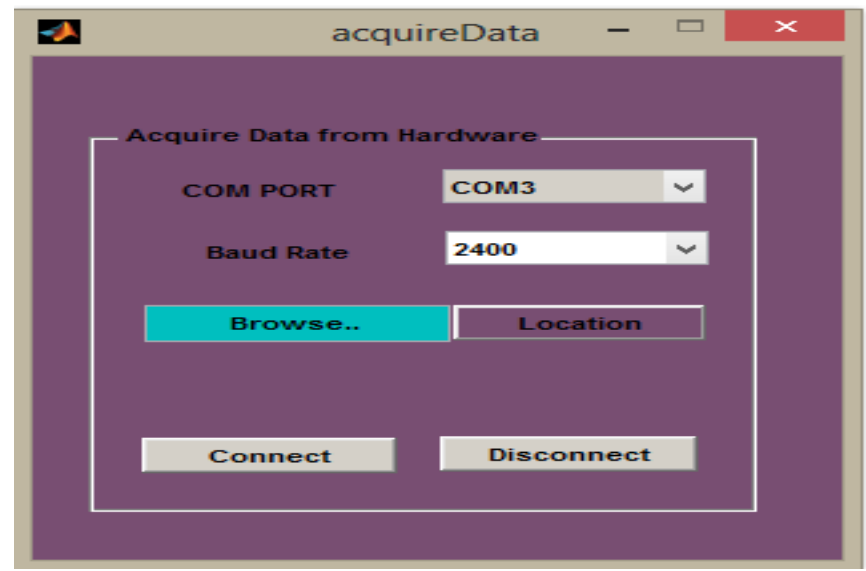




\subsection{Signal Analysis}

Fig. 5 Data Acquisition Window

Signals can be divided into stationary signals and non-stationary signals; the time domain sequence $x(n)$ is converted to frequency $X(k)$, the expression below is used to transform the signals from time domain to frequency domain.

$X(k)=\sum_{n=0}^{N-1} x(n) e^{-j 2 \pi k n / N} \quad k=0,1,2, \ldots, N-1$

The implementation of the DFT equation shown above requires a lot of memory and speed; hence it becomes imperative to use the enhanced fast Fourier transform algorithm (FFT), [1,11].

Finite-energy signals are usually characterized by their energy density spectrum using the auto-correlation function.

$P_{x x}(f)=\sum_{m=-(N-1)}^{N-1} r_{x x}(m) e^{-j 2 \pi f m}$

Where the auto-correlation function $r_{x x}$ is given as

$r_{x x}(m)=\frac{1}{N} \sum_{n=0}^{N-m-1} x^{*}(n) x(n+m) \quad 0 \leq m \leq N-1$

Where we can simply express $P_{x x}(f)$ as

$P_{x x}(f)=\frac{1}{N}\left|\sum_{n=0}^{N-1} x(n) e^{-j 2 \pi f n}\right|^{2}=\frac{1}{N}|X(f)|^{2}$

Where $X(f)$ is the Fourier transform of the sample sequence $x(n)$. This is usually referred to as the Periodogram [2,7].

The short time Fourier transform (STFT), gives a two dimensional representation of a continuous time signal. The STFT is given as

$\operatorname{STFT}\{x(t)\}=X(t, w)=\int_{-\infty}^{\infty} x(t) w(t-T) e^{-j w t} d t$

Where; $x(t)$ is the signal and $w(t)$ is the window function.

The most important advantage of using the STFT for analysis is the fact that it solves the problem of time resolution which the general Fourier transforms cannot handle. With the use of the STFT, it is possible to apply a window of width T over the signal, and sliding the window across the signal, within each of the sliding operation, the Fourier transform of the small portion of the signal is taken. This helps to provide a proper analysis of non-stationary signals. Hence giving rise to the Joint Time-Frequency Analysis (JTFA).

The interdependence of two sets of data or processes is established using correlation function as expressed in (6). The energy content of the data is defined using (7). However, the correlation function defined in (6) is magnitude dependent which can be normalized with the energy content of the data. The result is expressed by (8) as the coefficient of correlation.

$$
\begin{aligned}
& r_{11}(j)=\frac{1}{N}\left(\sum x(n) y(n+j)\right) \\
& r_{11}(j)=\frac{1}{N}\left(\sum x(n) x(n+j)\right) \\
& \rho(j)=\frac{\frac{1}{N}\left(\sum x(n) y(n+j)\right)}{\left(\sqrt{\frac{1}{N}\left(\sum x^{2}\right) \frac{1}{N}\left(\sum y^{2}\right)}\right)}
\end{aligned}
$$

\subsection{Filtering}

Digital Filter design is a significant part of signal processing [7,11]. The implementation of digital filters was performed on the module. The FFT power spectrum analysis allows the user to know the characteristics of the desired signal and that of the noise, and hence the type of filter that will be appropriate, which can be high pass, low pass, band pass or a stop band in nature. The characteristic of the filter which is expressed by the desired response includes the passband and stop band attenuations, and the transition width. These parameters are specified appropriately from the developed GUI. In addition, based on the desired response, the system automatically chooses the appropriate window function in the case of finite impulse response (FIR) filters. The spectrum of two sinusoidal signals mixed together is shown in Fig. 6. 


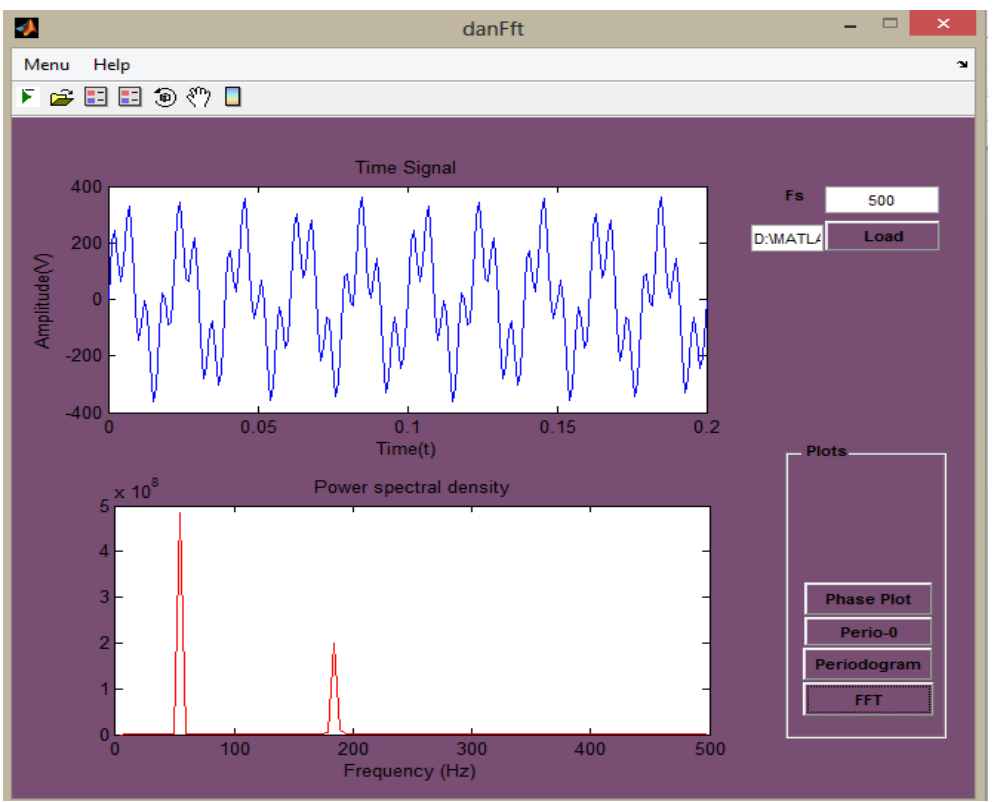

Fig. 6: Spectral Analysis Window

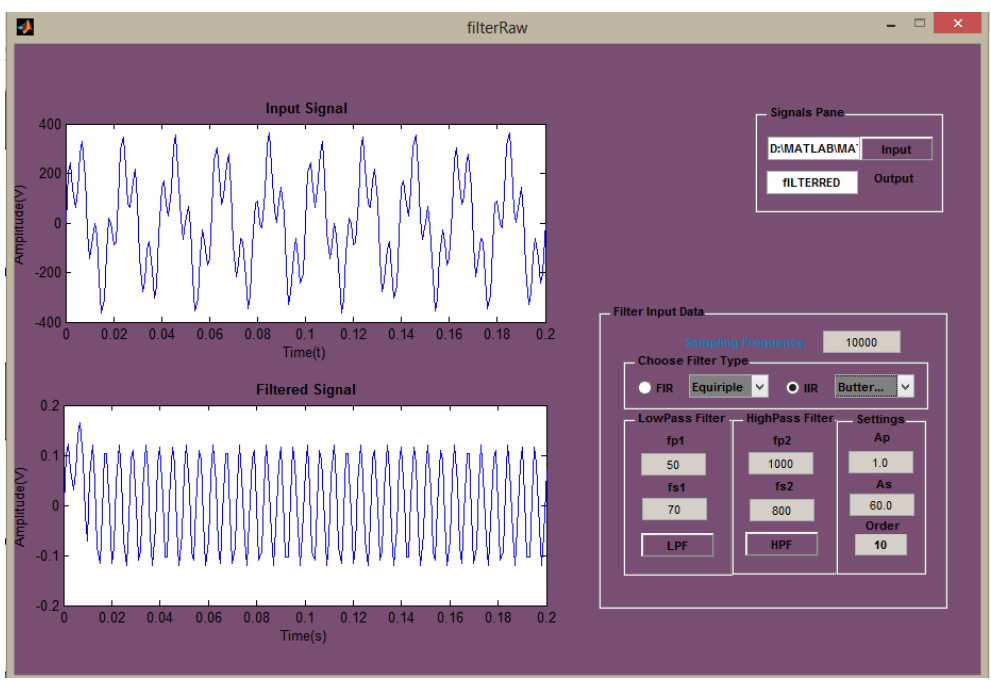

Fig. 7: Filter application window

The parameters to be specified are for the design of the appropriate FIR filter are:

- Passband attenuation - Ap

- Stopband Attenuation - As

- Sampling frequency $-\mathrm{Fs}$

- Passband frequency - Fp1 (lowpass filter)

- Stopband frequency - Fs1 (lowpass filter)

- Passband frequency - Fp2 (highpass filter)

- $\quad$ Stopband frequency - Fs2 (highpass filter)

In order to use this work for bandpass filtering operation, a highpass filtering is followed by lowpass filtering, with appropriate design parameters. The mixed sinusoid was passed through the designed Low Pass Filter and the result is shown in Fig. 7.

\subsection{Analysis of pre-recorded data}

This example illustrates how the developed software was used for the analysis of signals that have been pre-recorded form field, for instance, data from a remote sensor somewhere or data from a monitoring hardware from an industrial plant. This data is basically from sensors or any other external sources. 


\subsection{Generation of Signals}

This example illustrates the analysis of a software generated signal from the GUI. The signal is mixed with noise and then stored. A pure sine wave was generated and another signal which was harmonically related to the first signal was also generated, the two signals were then mixed, as shown in Fig. 8. The signal is then analyzed to determine the frequency components of the signal in order to determine the frequency of interest and the part to be filtered off. Since the signal is a stationary one, the FFT-based analysis is performed using the FFT capability of the developed system. Figure 8 shows the original signal and the corrupted signal.

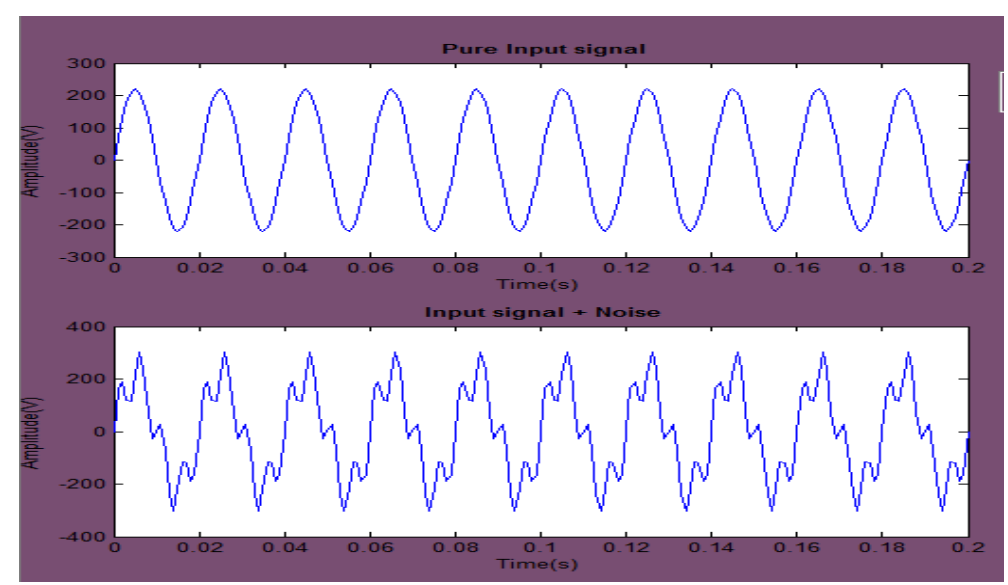

\subsection{Spectral Analysis}

Fig. 8: GUI generated data

The FFT analyzed spectrum is presented in Fig. 9. This result shows that the signal consists of two different frequency components centered at $100 \mathrm{~Hz}$ and $350 \mathrm{~Hz}$. The result concludes that the spectral components of any signal can be revealed by the developed module.

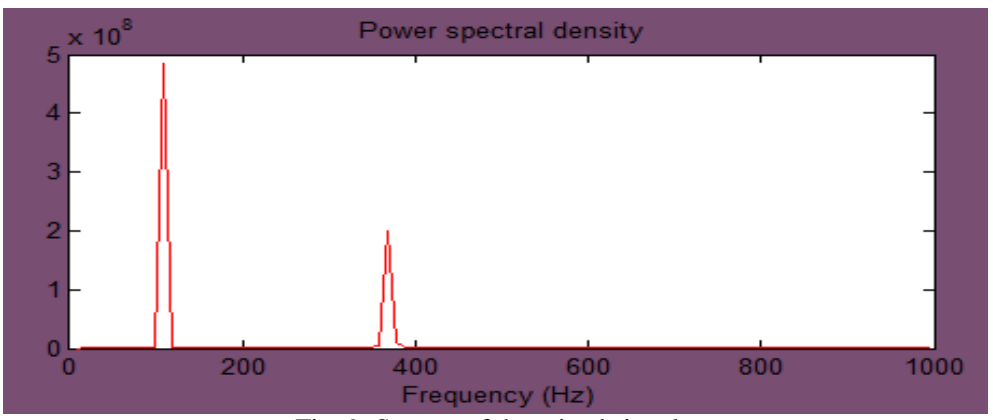

\subsubsection{Removal of Unwanted Signal}

Fig. 9: Spectra of the mixed signals

In this sub-section, the module was used to remove the unwanted signal from the harmonically related signal of Fig. 9.The parameters that include the sampling frequency, cut-off frequency; maximum passband attenuation, stopband attenuation and the filter type were set from the filtering window of the module. Since the filtering method employs the window method, the selection of window type is made automatically by the system based on the attenuation requirements. The filtered signal is as shown in Fig. 10.

\subsection{Correlation}

The degree of interdependence of two sets of data or process can be evaluated using correlation. The module was used to carry out the following on the data:

(a) Plot the correlation coefficient at different lags

(b) Determine the correlation of a two different signals

(c) Determine the period of a periodic signal buried in random noise

The results are presented in Fig11. Fig. 12 and Fig. 13 


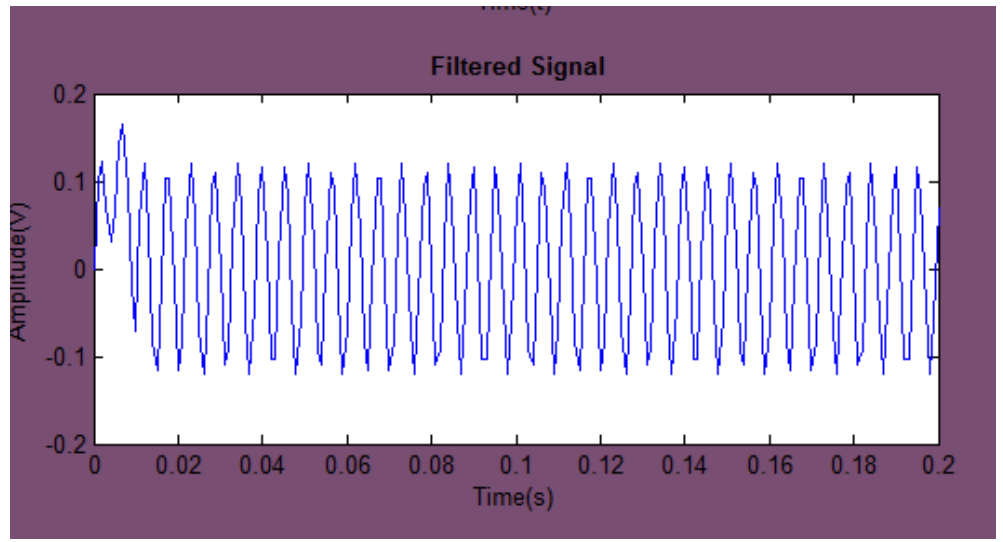

Fig. 10: Filtered Signal

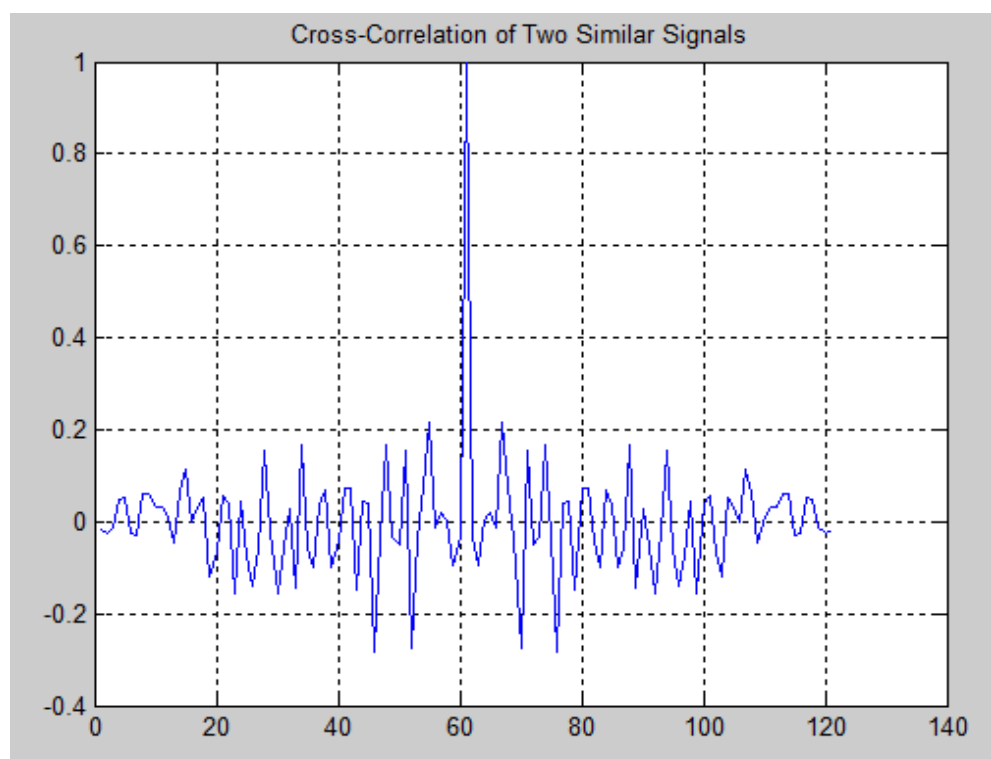

Fig.11: Correlation coefficient at different lags for similar signals with phase different

The results show that the signal are shifted in phase and the highest correlation coefficient obtained after many lags is shown in Fig 11

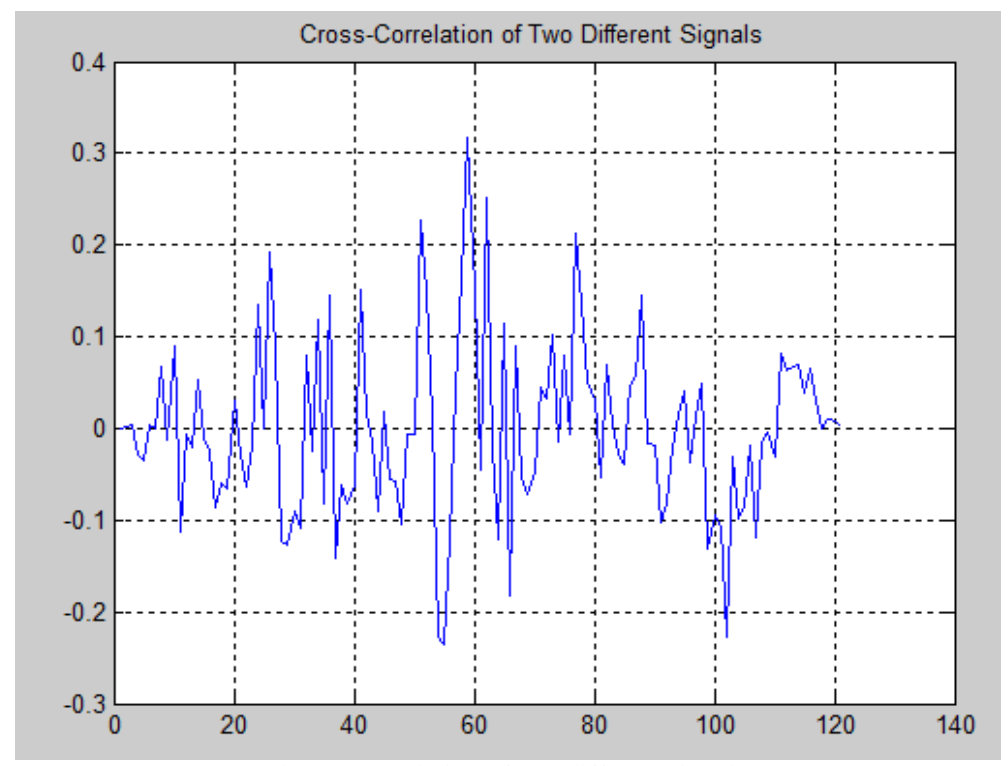

Fig. 12: correlation of two different signals 
The result in Fig. 12 shows the correlation of two signals that are not correlated

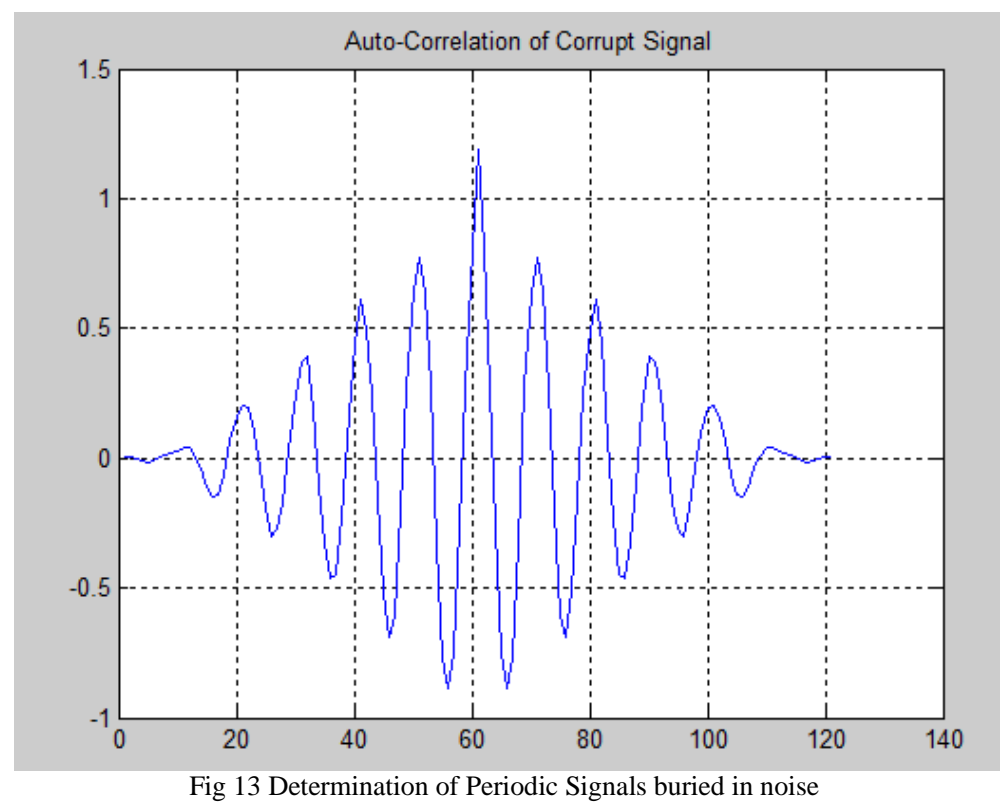

Fig. 13 shows that the periodicity of a signal buried in noise can be determined by autocorrelation.

\section{Conclusion}

The development of MATLAB-based GUI for PIC microcontroller projects was presented in this paper. The module offers embedded systems developer a unique platform to acquire and process signals. The module performed to the design specifications. The result shows that both stationary and non-stationary signals can be analyzed by the module. The developed system provides interactive environment useful for the designed objectives. The results obtained from the experimentation carried out show that the module is suitable for the purpose of solving problem encountered in the teaching of DSP. This work serves as a veritable tool for students and educators in Electronics and Signal Processing. The objective of developing a module that will be useful for the teaching of DSP in Nigerian University at affordable cost was achieved.

\section{References}

[1] Online: http://www.microchip.com/1010/index.htm, website of Microchip Technology, Inc.

[2] D. W. Smith, PIC in Practice, (2003), Newnes, Oxford, U.K.,.

[3] Monson H. Harris, Digital Signal Processing, (1999), Schaum's Outline, McGraw-Hill, New York,

[4] S.-H. Lee, Y.-F. Li, and V. Kapila, "Development of a Matlab-Based Graphical User Interface for PIC Microcontroller Projects," Proceedings of the American Society of Engineering Education Conference, Salt Lake City, UT, Session, (2004), pp2220.

[5] Y. F. Li, S. Harari, H. Wong, and V. Kapila, "Matlab-Based Graphical User Interface Development for Basic Stamp 2 Microcontroller Projects," Proceedings of the American Control Conference, Boston, MA, (2004), pp. 3233-3238,

[6] Online. http://www.usbpicprog.org

[7] Proakis, Jhon G. and Manolakis Dimitris G., Digital Signal Processing: Principles, Algorithms and Applications, Pearson Education Inc., 2007.

[8] www.mathworks.com

[9] Online: http://www.microchip.com/1010/suppdoc/appnote/index.htm, website of Microchip Technology, Inc., (access link for application notes, code examples, and templates).

[10] www.nuc.ng.org

[11] Oppenheim, A.V., and R.W. Schafer, Discrete-Time Signal Processing, Prentice-Hall, 1989, pp. 447-448 